\title{
Probing pseudo Nambu-Goldstone boson dark matter at loop level
}

\author{
Koji Ishiwata ${ }^{a}$ and Takashi Toma ${ }^{b}$ \\ ${ }^{a}$ Institute for Theoretical Physics, Kanazawa University, \\ Kanazawa 920-1192, Japan \\ ${ }^{b}$ Physik-Department T30d, Technische Universität München, \\ James-Franck-Straße, D-85748 Garching, Germany \\ E-mail: ishiwata@hep.s.kanazawa-u.ac.jp, takashi.toma@tum.de
}

ABSTRACT: In the standard model extended by a single complex scalar boson with a softly broken global U(1) symmetry, a pseudo Nambu-Goldstone boson becomes a candidate for dark matter. In this paper, we discuss the direct detection of the pseudo Nambu-Goldstone boson dark matter. Since the tree-level amplitude for dark matter-nucleon scattering vanishes, higher order quantum corrections for the amplitude should be taken into account. We perform the calculation at the next-to-leading order in QCD in a systematic manner.

KEywords: Beyond Standard Model, Cosmology of Theories beyond the SM, Global Symmetries

ARXIV EPRINT: 1810.08139 


\section{Contents}

1 Introduction 1

2 The model 2

3 Scattering cross section 4

3.1 Formalism 5

3.2 Wilson coefficients 6

3.3 Numerical results 7

$\begin{array}{llr}4 & \text { Conclusion } & 9\end{array}$

A Loop functions $\quad 10$

A.1 Tadpoles 11

$\begin{array}{lll}\text { A.2 Self-energies } & 11\end{array}$

A.3 Vertex corrections 11

$\begin{array}{lll}\text { A.4 Box and triangle diagrams } & 12\end{array}$

\section{Introduction}

Although it is well-known that non-baryonic dark matter (DM) exists in the universe, nature of dark matter is still a mystery of the universe except the fact that its relic abundance occupies about $26 \%$ of the total energy density of the universe [1, 2]. A prominent candidate for DM is a stable and non-relativistic particle that has the weak scale interactions. In this case, the annihilation rate determining its relic abundance is closely correlated with scattering rates with the Standard Model (SM) particles and production rates at collider experiments through the crossing symmetry.

Direct detection experiments of cold dark matter explores scattering events between DM and nuclei. So far, any viable signal of DM has not been found even in recent tonscale detector experiments, which leads to the bounds on the interaction between DM and nucleon. The most stringent upper bound on the spin independent (SI) elastic scattering cross section between DM and nucleon is given by the XENON1T Collaboration [3], which is, for example, $4.1 \times 10^{-47} \mathrm{~cm}^{2}$ at the DM mass of $30 \mathrm{GeV}$ at $90 \%$ confidence level.

The current null results of direct signal of DM motivate us to consider a framework where the interactions between DM and nucleon are suppressed in non-relativistic limit. One of the ideas is to consider a pseudo Nambu-Goldstone DM [4] or a pseudo scalar portal fermionic DM [5-7]. In the former case all the interactions between DM and SM particles are described by derivative couplings at the tree level, while in the latter case the DM spinor product for elastic scattering is proportional to the DM velocity in non-relativistic 
limit. As a result, the elastic scattering amplitude is necessarily suppressed by the small DM velocity at tree level, thus these DM candidates can naturally be consistent with the strong constraints from the current direct detection experiments.

In this paper we consider a pseudo Nambu-Goldstone DM proposed in ref. [4], and study the possibility to detect it directly. As stated above, the elastic scattering amplitude for direct detection is suppressed at the tree level in this model, and it can be exactly zero in non-relativistic limit. However, the scattering amplitude is expected to be finite at the loop level. We will perform the calculation at one-loop level for non-QCD part and at the next-to-leading order level in QCD based on the formalism given in ref. [8] where the scattering processes with gluon in nucleon, which are sometimes missed in the literature, are systematically taken into account. Although the next-to-leading order calculation in QCD is not necessary for a rough evaluation, it gives $\mathcal{O}(10 \%)$ corrections in the amplitude and the theoretical uncertainty regarding perturbative QCD calculation is reduced significantly. We explore a parameter space consistent with the observed DM relic abundance, the SM Higgs boson decay, and the perturbative unitarity bound. We also compare the predicted elastic scattering cross section with the sensitivity of the future direct detection experiment DARWIN [9].

\section{The model}

We consider the SM augmented by a complex scalar field $S$ with a softly broken global $\mathrm{U}(1)$ symmetry. The model is invariant under the transformation $S \rightarrow e^{i \alpha} S$ with a real constant $\alpha$ except for the soft breaking term. The scalar potential of the model is given by

$$
\mathcal{V}=-\frac{\mu_{\mathrm{H}}^{2}}{2}|H|^{2}-\frac{\mu_{\mathrm{S}}^{2}}{2}|S|^{2}+\frac{\lambda_{\mathrm{H}}}{2}|H|^{4}+\lambda_{\mathrm{HS}}|H|^{2}|S|^{2}+\frac{\lambda_{\mathrm{S}}}{2}|S|^{4}-\left(\frac{\mu_{\mathrm{S}}^{\prime 2}}{4} S^{2}+\text { H.c. }\right),
$$

where $H$ is the $\mathrm{SU}(2)_{L}$ Higgs doublet which couples to the SM particles. The last term corresponds to the soft breaking term of the global U(1) symmetry. Due to the tachyonic mass terms for the scalar fields, both $H$ and $S$ acquire vacuum expectation values (VEVs) in a wide range of the parameter space, which is the situation we are interested in. Then these fields are expanded around the vacuum as

$$
H=\left(\begin{array}{c}
G^{+} \\
\frac{1}{\sqrt{2}}\left(v+h+i G^{0}\right)
\end{array}\right), \quad S=\frac{v_{s}+s+i \chi}{\sqrt{2}},
$$

where $G^{+}$and $G^{0}$ are the Nambu-Goldstone bosons associated with the electroweak symmetry breaking, $v(\simeq 246 \mathrm{GeV})$ and $v_{s}$ are the VEVs for $H$ and $S$, respectively. $h$ and $s$ are the $\mathrm{CP}$ even scalar fields while $\chi$ is the CP odd scalar field which is the would-be Nambu-Goldstone boson. Due to the soft breaking term of the global U(1) symmetry, non-zero mass for $\chi$ arises. Even after the symmetry breaking, a $\mathbb{Z}_{2}$ symmetry remains, which stabilizes $\chi$ and makes it a candidate for DM.

Due to the symmetry breaking, the CP even states $h$ and $s$ mix via the Higgs portal coupling $\lambda_{\mathrm{HS}}$. We will derive the mass eigenstates $h_{1}$ and $h_{2}$ at one-loop level. As mentioned in Introduction, $\chi-q$ scattering amplitude vanishes at the tree level. Therefore, one-loop 
corrections are necessary for studying direct detection of $\chi$ DM. To this end, we execute the calculation following ref. [10]. In the literature, the inverse propagators for the scalars are calculated diagramatically. Then the mass matrices for the CP odd and even sectors are defined by taking zero external momenta. That corresponds to the one obtained from the effective potential. Then the mass eigenstates are given by diagonalizing the mass matrices. In the following calculation we adopt Landau gauge and $\overline{\mathrm{MS}}$ renormalization scheme as in the literature. All couplings, scalar fields, and VEVs are renormalized values. We will see that the renormalization scale dependence is cancelled in the amplitude as expected.

The mass matrices for the $\mathrm{CP}$ even and $\mathrm{CP}$ odd sectors are given by

$$
\begin{aligned}
M_{\text {even }}^{2} & =\left(\begin{array}{cc}
\lambda_{\mathrm{H}} v^{2}+\frac{T_{h}}{v} & \lambda_{\mathrm{HS}} v v_{s} \\
\lambda_{\mathrm{HS}} v v_{s} & \lambda_{\mathrm{S}} v_{s}^{2}+\frac{T_{s}}{v_{s}}
\end{array}\right) \equiv\left(\begin{array}{cc}
M_{h h}^{2} & M_{h s}^{2} \\
M_{h s}^{2} & M_{s s}^{2}
\end{array}\right), \\
M_{\mathrm{odd}}^{2} & =\left(\begin{array}{cc}
\frac{T_{h}}{v} & 0 \\
0 & \mu_{\mathrm{S}}^{\prime 2}+\frac{T_{s}}{v_{s}}
\end{array}\right) \equiv\left(\begin{array}{cc}
M_{G^{0} G^{0}}^{2} & 0 \\
0 & M_{\chi \chi}^{2}
\end{array}\right),
\end{aligned}
$$

where $T_{h}$ and $T_{s}$ are renormalized tadpoles for $h$ and $s$, which satisfy the stationary conditions:

$$
\begin{array}{r}
\mu_{\mathrm{H}}^{2}-\lambda_{\mathrm{H}} v^{2}-\lambda_{\mathrm{HS}} v_{s}^{2}+\frac{2 T_{h}}{v}=0, \\
\mu_{\mathrm{S}}^{2}-\lambda_{\mathrm{S}} v_{s}^{2}-\lambda_{\mathrm{HS}} v^{2}+\mu_{\mathrm{S}}^{\prime 2}+\frac{2 T_{s}}{v_{s}}=0 .
\end{array}
$$

Then, the inverse propagators are given as

$$
\begin{aligned}
\Gamma_{i j}\left(p^{2}\right) & =\delta_{i j} p^{2}-M_{i j}^{2}+\Pi_{i j}\left(p^{2}\right) \\
& =\delta_{i j} p^{2}-\bar{M}_{i j}^{2}+\Delta \Pi_{i j}^{2}\left(p^{2}\right),
\end{aligned}
$$

where indices $i, j$ represent $h, s$ (or $\left.h_{1}, h_{2}\right), G^{0}, \chi$, and $\Pi_{i j}\left(p^{2}\right)$ correspond to the renormalized self-energies with the external lines $i$ and $j$. The concrete expressions for $\Pi_{i j}\left(p^{2}\right)$ are collected in appendix. Here we have introduced the quantities $\Delta \Pi_{i j}\left(p^{2}\right)$ defined by

$$
\Delta \Pi_{i j}\left(p^{2}\right) \equiv \Pi_{i j}\left(p^{2}\right)-\Pi_{i j}(0),
$$

and the definition of $\bar{M}_{i j}^{2}$ in eq. (2.7) follows accordingly. Note that $\bar{M}_{i j}^{2}$ correspond to the mass matrices derived from the effective potential, i.e., zero external momenta. Since the mass matrix $M_{\text {odd }}^{2}$ is diagonal, the physical (pole) masses for the CP odd fields are simply given by $\Gamma_{G^{0} G^{0}}(0)=0$ and $\Gamma_{\chi \chi}\left(m_{\chi}^{2}\right)=0$ where $m_{\chi}$ is the pole mass of $\chi$, i.e.,

$$
\begin{aligned}
0 & =M_{G^{0} G^{0}}^{2}-\Pi_{G^{0} G^{0}}(0), \\
m_{\chi}^{2} & =M_{\chi \chi}^{2}-\Pi_{\chi \chi}\left(m_{\chi}^{2}\right) .
\end{aligned}
$$

The CP even sector, on the other hand, needs to be diagonalized. Following ref. [10], we derive an eigenstate basis with one-loop correction by diagonalizing $\bar{M}_{i j}^{2}$. Using the equations given above, $\bar{M}_{i j}^{2}$ in the $\mathrm{CP}$ even sector are rewritten as

$$
\begin{aligned}
\bar{M}_{h h}^{2} & =\lambda_{\mathrm{H}} v^{2}+\Delta_{11}, \\
\bar{M}_{h s}^{2} & =\lambda_{\mathrm{HS}} v v_{s}+\Delta_{12}, \\
\bar{M}_{s s}^{2} & =\lambda_{\mathrm{S}} v_{s}^{2}-\mu_{S}^{\prime 2}+m_{\chi}^{2}+\Delta_{22}+\Delta \Pi_{\chi \chi}\left(m_{\chi}^{2}\right),
\end{aligned}
$$


where

$$
\begin{aligned}
\Delta_{11} & \equiv \Pi_{G^{0} G^{0}}(0)-\Pi_{h h}(0), \\
\Delta_{12} & \equiv-\Pi_{h s}(0), \\
\Delta_{22} & \equiv \Pi_{\chi \chi}(0)-\Pi_{s s}(0) .
\end{aligned}
$$

Then the mixing angle for the diagonalization is obtained by

$$
O^{T}\left(\begin{array}{cc}
\bar{M}_{h h}^{2} & \bar{M}_{h h}^{2} \\
\bar{M}_{h s}^{2} & \bar{M}_{s s}^{2}
\end{array}\right) O=\left(\begin{array}{cc}
\bar{m}_{h_{1}}^{2} & 0 \\
0 & \bar{m}_{h_{2}}^{2}
\end{array}\right),
$$

with

$$
O=\left(\begin{array}{cc}
\cos \theta & \sin \theta \\
-\sin \theta & \cos \theta
\end{array}\right), \quad \tan 2 \theta=-\frac{2 \bar{M}_{h s}^{2}}{\bar{M}_{h h}^{2}-\bar{M}_{s s}^{2}} .
$$

The eigenstates $h_{1} h_{2}$ are then given by $\left(h_{1}, h_{2}\right)^{T}=O^{T}(h, s)^{T}$. We define $h_{1}$ as the lighter field, which is identified as the observed Higgs boson. Their physical masses are then derived straightforwardly as

$$
m_{h_{i}}^{2}=\bar{m}_{h_{i}}^{2}-\Delta \Pi_{h_{i} h_{i}}\left(m_{h_{i}}^{2}\right) .
$$

Using the mass eigenstates, the scalar potential can be expanded around the VEVs. In the mass eigenstate basis, the coefficients for scalar cubic and quartic couplings, which are relevant for our discussion, are expressed as

$$
\begin{aligned}
\mathcal{V} \supset & \sum_{i}\left(c_{\chi \chi h_{i}} \chi^{2} h_{i}+c_{G^{0} G^{0} h_{i}} G^{0^{2}} h_{i}\right)+\sum_{i \leq j \leq k} c_{h_{i} h_{j} h_{k}} h_{i} h_{j} h_{k} \\
& +d_{\chi \chi \chi \chi} \chi^{4}+d_{G^{0} G^{0} G^{0} G^{0} G^{0^{4}}+d_{\chi \chi G^{0} G^{0}} \chi^{2} G^{0}} \\
& +\sum_{i \leq j}\left(d_{\chi \chi h_{i} h_{j}} \chi^{2} h_{i} h_{j}+d_{G^{0} G^{0} h_{i} h_{j}} G^{0^{2}} h_{i} h_{j}\right)+\sum_{i \leq j \leq k \leq m} d_{h_{i} h_{j} h_{k} h_{m}} h_{i} h_{j} h_{k} h_{m},
\end{aligned}
$$

where $i, j, k, m$ are 1 or 2 . Additionally, the Yukawa couplings to quarks are given by

$$
\mathcal{L}_{\text {Yukawa }} \supset-\sum_{i} y_{q q h_{i}} h_{i} \bar{q} q
$$

where $q$ are quarks.

\section{Scattering cross section}

In this section we compute the SI cross section of $\chi$ DM with nucleon. To avoid confusing readers, we clarify some terminology related to our calculation. We will perform the calculation literally at one-loop level for non-QCD related part. For QCD part, on the other hand, the amplitude is derived at the next-to-leading order (NLO) level. Throughout this paper we use the term $N L O$ or leading order $(L O)$ in terms of order of QCD strong coupling $\alpha_{s}$. For example, $L O$ contains one-loop diagrams for $\chi-g$ scattering. However, the gluon contributions to the effective scalar coupling are $\mathcal{O}\left(\alpha_{s}^{0}\right)[8,11,12]$. That is why we use the term $L O$ for such $\chi-g$ processes, and similar discussion is applied for $N L O$. As we will see, the gluon contributions become important in some parameter space. 


\subsection{Formalism}

We briefly summarize the formalism for the calculation of the SI scattering cross section of a real scalar DM with nucleon based on refs. [8, 13]. Using the formalism in ref. [8], we calculate the scattering amplitude at the NLO in QCD.

The effective Lagrangian relevant for the scattering process is

$$
\mathcal{L}_{\text {eff }}=\sum_{i=q, G} C_{\mathrm{S}}^{i} \mathcal{O}_{\mathrm{S}}^{i}+\sum_{i=q, G} C_{\mathrm{T}}^{i} \mathcal{O}_{\mathrm{T}}^{i}
$$

where $C_{\mathrm{S}}^{i}$ and $C_{\mathrm{T}}^{i}$ are the Wilson coefficients and the operators $\mathcal{O}_{\mathrm{S}}^{i}$ and $\mathcal{O}_{\mathrm{T}}^{i}$ are given by

$$
\begin{aligned}
\mathcal{O}_{\mathrm{S}}^{q} & \equiv m_{q} \chi^{2} \bar{q} q \\
\mathcal{O}_{\mathrm{S}}^{G} & \equiv \frac{\alpha_{s}}{\pi} \chi^{2} G_{\mu \nu}^{a} G^{a \mu \nu}, \\
\mathcal{O}_{\mathrm{T}}^{i} & \equiv \frac{1}{m_{\chi}^{2}} \chi^{i \partial^{\mu} i \partial^{\nu} \chi \mathcal{O}_{\mu \nu}^{i}} .
\end{aligned}
$$

Here $G_{\mu \nu}^{a}$ represents the field strength tensor of gluon field and the quark masses are denoted as $m_{q}$. The operators $\mathcal{O}_{\mu \nu}^{q}$ and $\mathcal{O}_{\mu \nu}^{G}$ are the twist-2 operators of quarks and gluon, respectively, which are defined by

$$
\begin{aligned}
\mathcal{O}_{\mu \nu}^{q} & \equiv \frac{1}{2} \bar{q} i\left(D_{\mu} \gamma_{\nu}+D_{\nu} \gamma_{\mu}-\frac{1}{2} g_{\mu \nu} \not D\right) q, \\
\mathcal{O}_{\mu \nu}^{G} & \equiv G_{\mu}^{a \rho} G_{\nu \rho}^{a}-\frac{1}{4} g_{\mu \nu} G_{\rho \sigma}^{a} G^{a \rho \sigma}
\end{aligned}
$$

with $D_{\mu}$ the covariant derivative. Then the SI scattering cross section of $\chi$ with nucleon $N$ is obtained as $[8,13]$

$$
\sigma_{\mathrm{SI}}^{N}=\frac{1}{\pi}\left(\frac{m_{N}}{m_{\chi}+m_{N}}\right)^{2}\left|f_{\mathrm{scalar}}^{N}+f_{\mathrm{twist} 2}^{N}\right|^{2}
$$

where $m_{N}$ is the nucleon mass, and $f_{\text {scalar }}^{N}$ and $f_{\text {twist2 }}^{N}$ are given by

$$
\begin{aligned}
\frac{f_{\text {scalar }}^{N}}{m_{N}} & =\sum_{q=u, d, s} C_{\mathrm{S}}^{q}\left(\mu_{\mathrm{had}}\right) f_{T q}^{N}-\frac{8}{9} C_{\mathrm{S}}^{G}\left(\mu_{\mathrm{had}}\right) f_{T g}^{N}, \\
\frac{f_{\mathrm{twist} 2}^{N}}{m_{N}} & =\frac{3}{4} \sum_{q}\left[C_{\mathrm{T}}^{q}\left(m_{Z}\right)\left[q^{N}\left(2 ; m_{Z}\right)+\bar{q}^{N}\left(2 ; m_{Z}\right)\right]-C_{\mathrm{T}}^{G}\left(m_{Z}\right) g^{N}\left(2 ; m_{Z}\right)\right] .
\end{aligned}
$$

Here $f_{T q}^{N}, f_{T g}^{N}, q^{N}\left(2 ; m_{Z}\right), \bar{q}^{N}\left(2 ; m_{Z}\right)$, and $g^{N}\left(2 ; m_{Z}\right)$ are the matrix elements of the effective operators in nucleon state. $\mu_{\text {had }}$ is the hadronic scale (i.e., around $1 \mathrm{GeV}$ ), and $m_{Z}$ is the $Z$ boson mass. The numerical values for these quantities are given in ref. $[8]^{1}$ based on the QCD lattice simulation [14, 15] and CETEQ-Jefferson Lab collaboration [16]. As we will see, the contribution to the twist-2 type operators is negligibly small. Therefore, the SI cross section is determined by the scalar-type interactions. 
(i)

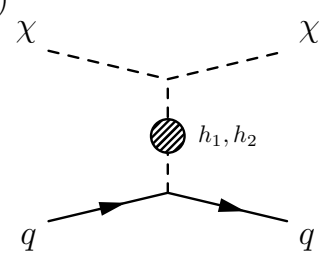

(iii)

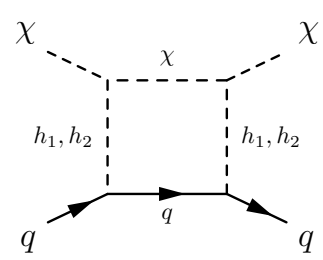

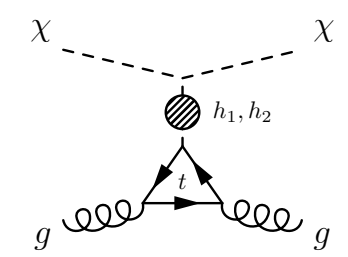

(ii)

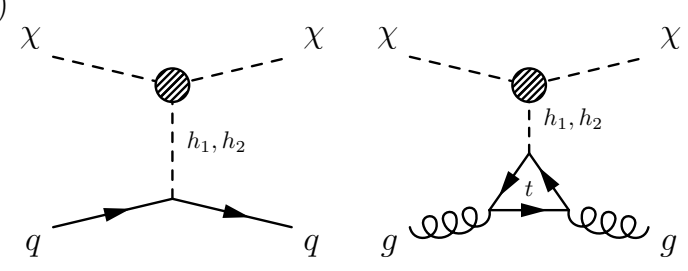

Figure 1. Feynman diagrams for $\chi-q$ and $\chi-g$ scattering processes.

\subsection{Wilson coefficients}

First of all, we derive the effective Lagrangian from full theory by matching at the weak scale denoted as $\mu_{W} \simeq m_{Z}$. As described in Introduction, the tree-level amplitudes for $\chi q \rightarrow \chi q$ are cancelled in the non-relativistic limit. Therefore loop-level calculations are necessary to evaluate the scattering amplitude in the limit. There are three types of diagrams shown in figure 1; (i) Self-energies, (ii) Vertex corrections, and (iii) Box and triangle diagrams. ${ }^{2}$

The most part of the computation for the diagrams (i) has already been done in the previous section. Let us apply the results to the scattering process. The diagrams (i) give rise to scalar-type interactions. The matching at the weak scale gives

$$
\begin{aligned}
&\left.C_{\mathrm{S}}^{q}\left(\mu_{W}\right)\right|_{\text {self }}=-\frac{1}{2 m_{h_{1}}^{2} m_{h_{2}}^{2} v v_{s}} {\left[\Delta_{12}\left(\sin ^{2} \theta m_{h_{1}}^{2}+\cos ^{2} \theta m_{h_{2}}^{2}\right)\right.} \\
&\left.+\tilde{\Delta}_{22} \sin \theta \cos \theta\left(m_{h_{1}}^{2}-m_{h_{2}}^{2}\right)\right], \\
&\left.C_{\mathrm{S}}^{G}\left(\mu_{W}\right)\right|_{\text {self }}=-\left.\frac{1}{12}\left[1+\frac{11 \alpha_{s}}{4 \pi}\right] C_{\mathrm{S}}^{q}\left(\mu_{W}\right)\right|_{\text {self }},
\end{aligned}
$$

for $q=u, d, s, c, b$ where $\Delta_{12}$ is given in eq. (2.15), and $\tilde{\Delta}_{22}$ is given by

$$
\tilde{\Delta}_{22}=\frac{T_{s}}{v_{s}}-\Pi_{s s}(0)
$$

The computation of the diagrams (ii) is rather simple. These diagrams give vertex corrections to the $\chi-\chi-h_{i}$ couplings. Denoting them as $\Delta c_{\chi \chi h_{i}}$ (collected in appendix), the

\footnotetext{
${ }^{1}$ To be strict, $f_{T q}^{N}$ corresponds to $f_{T q}^{(N)}$ in ref. [8] and so on. We have additionally introduced $f_{T g}^{N}$ defined as $(-8 / 9) f_{T g}^{N} \equiv\left\langle N\left|\frac{\alpha_{s}}{\pi} G_{\mu \nu}^{a} G^{a \mu \nu}\right| N\right\rangle / m_{N}$ evaluated at three flavors, which leads to $f_{T g}^{N}=1-\sum_{q=u, d, s} f_{T q}^{N}+$ $\mathcal{O}\left(\alpha_{s}\right)$.

${ }^{2}$ The NLO diagrams for QCD part are not depicted for simplicity, but these are taken into account in the numerical study.
} 
Wilson coefficients are obtained as

$$
\begin{aligned}
\left.C_{\mathrm{S}}^{q}\left(\mu_{W}\right)\right|_{\mathrm{vert}} & =\frac{1}{m_{h_{1}}^{2} m_{h_{2}}^{2} v}\left[\Delta c_{\chi \chi h_{1}} \cos \theta m_{h_{2}}^{2}+\Delta c_{\chi \chi h_{2}} \sin \theta m_{h_{1}}^{2}\right], \\
\left.C_{\mathrm{S}}^{G}\left(\mu_{W}\right)\right|_{\mathrm{vert}} & =-\left.\frac{1}{12}\left[1+\frac{11 \alpha_{s}}{4 \pi}\right] C_{\mathrm{S}}^{q}\left(\mu_{W}\right)\right|_{\mathrm{vert}},
\end{aligned}
$$

for $q=u, d, s, c, b$. It is noted that, although both $\left.C_{\mathrm{S}}^{q}\left(\mu_{W}\right)\right|_{\text {self }}$ and $\left.C_{\mathrm{S}}^{q}\left(\mu_{W}\right)\right|_{\text {vert }}$ have the renormalization scale dependence, the dependence is cancelled in $\left.C_{\mathrm{S}}^{q}\left(\mu_{W}\right)\right|_{\text {self }}+\left.C_{\mathrm{S}}^{q}\left(\mu_{W}\right)\right|_{\text {vert }}$ as expected.

The $\chi-q$ scattering process drawn in the diagrams (iii) gives both scalar and twist-2 type contributions. However, the resultant Wilson coefficients are proportional to $y_{q q h_{i}}^{2} \propto m_{q}^{2}$ ( $q=u, d, s, c, b$ ), thus they are negligibly small. For the $\chi-g$ scattering, on the other hand, the top loop diagram should be taken into account since there is no such suppression. They can be calculated easily in Fock-Schwinger gauge (see appendix for details) and the resultant expressions are

$$
\left.C_{\mathrm{S}}^{G}\left(\mu_{W}\right)\right|_{\mathrm{box}+\mathrm{tri}}=\frac{1}{m_{\chi}^{4}} \sum_{i \leq j} y_{t t h_{i}} y_{t t h_{j}}\left[c_{\chi \chi h_{i}} c_{\chi \chi h_{j}} J_{\mathrm{box}}^{i j}+m_{\chi}^{2} d_{\chi \chi h_{i} h_{j}} J_{\mathrm{tri}}^{i j}\right] .
$$

We refer to the terms proportional to $J_{\text {box }}^{i j}$ and $J_{\text {tri }}^{i j}$ as 'box' type and 'triangle' type, respectively. Note that there is no $m_{\chi}$ dependence in $J_{\text {tri }}^{i j} / m_{\chi}^{2}$, which is obvious from the corresponding diagrams. It is found numerically that the contribution to the amplitude from the box-type diagrams is much smaller than that from the triangle-type diagrams in the parameter space we are interested in. Here we have ignored NLO contributions and we will treat it as a theoretical uncertainty as in ref. [8]. This is because it is expected to be suppressed compared to the other NLO contributions.

To summarize, the weak scale matching gives

$$
\begin{aligned}
& C_{\mathrm{S}}^{q}\left(\mu_{W}\right)=\left.C_{\mathrm{S}}^{q}\left(\mu_{W}\right)\right|_{\text {self }}+\left.C_{\mathrm{S}}^{q}\left(\mu_{W}\right)\right|_{\text {vert }}, \\
& C_{\mathrm{S}}^{G}\left(\mu_{W}\right)=\left.C_{\mathrm{S}}^{G}\left(\mu_{W}\right)\right|_{\text {self }}+\left.C_{\mathrm{S}}^{G}\left(\mu_{W}\right)\right|_{\text {vert }}+\left.C_{\mathrm{S}}^{G}\left(\mu_{W}\right)\right|_{\text {box }+ \text { tri }} .
\end{aligned}
$$

The Wilson coefficients at the hadronic scale $\mu_{\text {had }}$ are obtained by the renormalization group equations, along with the matching at bottom and charm mass scales, consistently at the NLO in QCD [8].

\subsection{Numerical results}

Now we are ready to show the numerical results. The SI cross section of $\chi \mathrm{DM}$ with proton is plotted in figure 2. We have computed the SI cross section at the NLO in QCD. For comparison, the results at the LO in QCD and the results obtained by an approximate expression for $f_{\text {scalar }}^{N}$ given by

$$
f_{\text {scalar }}^{N} / m_{N} \approx\left[\frac{2}{9}+\frac{7}{9} \sum_{q=u, d, s} f_{T q}^{N}\right] C_{\mathrm{S}}^{q}\left(\mu_{W}\right)-\left.\frac{8}{9} f_{T g}^{N} C_{\mathrm{S}}^{G}\left(\mu_{W}\right)\right|_{\text {box }+ \text { tri }},
$$



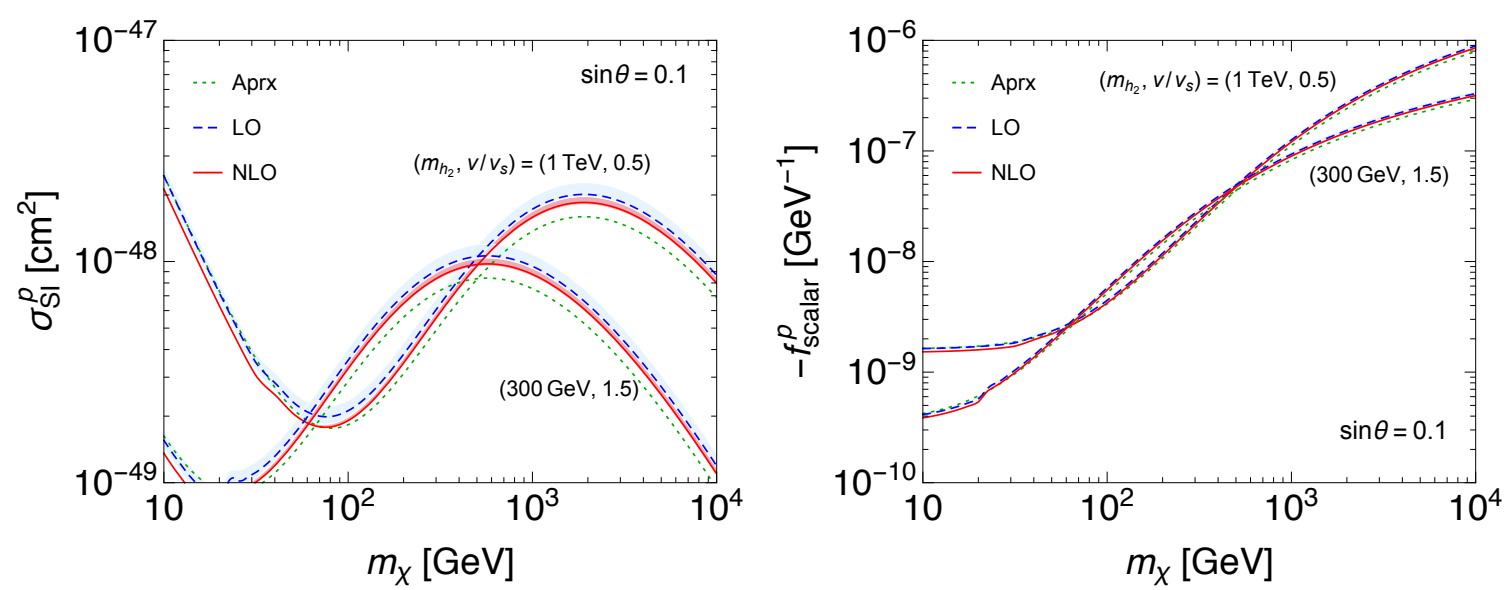

Figure 2. (Left) Spin-independent cross section of DM with proton for two sample parameter points, $\left(m_{h_{2}}, v / v_{s}\right)=(300 \mathrm{GeV}, 1.5)$ and $(1 \mathrm{TeV}, 0.5)$, for $\sin \theta=0.1$. The numerical evaluations with the approximate expression eq. (3.15), LO and NLO in QCD are shown as 'Aprx' (green dotted), 'LO' (blue dashed), and 'NLO' (red solid). For LO and NLO results, the perturbative errors are also shown in shaded region. (Right) (Minus) scalar-type effective coupling $f_{\text {scalar }}^{p}$ for the same sample parameter points. The line legends are the same as left figure.

are shown as well. Here $f_{T g}^{N}$ is given at the LO. The deviations of the LO and the approximate results from the NLO results are both $\mathcal{O}(10 \%){ }^{3}$ Perturbative errors at the LO results are $\mathcal{O}(10 \%)$, which are reduced to a few $\%$ at the NLO level as expected. We have also checked that the errors due to the input parameters are $\mathcal{O}(10 \%)$, and that the uncertainty due to ignoring the NLO contribution in the diagrams (iii) is less than $1 \%$. The behavior of the cross section is understood from the scalar-type effective coupling $f_{\text {scalar }}^{N}$, which is plotted in the right of figure 2. It is found that in large $m_{\chi}$ region the contributions from the diagrams (iii) are subdominant in $f_{\text {scalar }}^{N}$, consequently, the effective coupling is determined by the diagrams (i) and (ii), i.e., the self-energies and vertex corrections that have a logarithmic dependence on $m_{\chi}$ for large $m_{\chi}$ (see eq. (A.16) in appendix). Thus $\left|f_{\text {scalar }}^{N}\right|$ increases as $\log m_{\chi}+$ const. In small $m_{\chi}$ region, on the contrary, the diagrams (iii) dominate over the diagrams (i) and (ii) which are suppressed by $m_{\chi}^{2}$ (see eq. (A.15) in appendix). This is why the cross section is not suppressed in small DM mass region. This turn-over in the effective coupling happens roughly around $m_{\chi} \sim \mathcal{O}\left(10-10^{3} \mathrm{GeV}\right)$.

Figure 3 shows various constraints on the model. The purple region is excluded by the constraint from the SM-like Higgs decay where the Higgs signal strength at the LHC $\mu=1.09_{-0.10}^{+0.11}$ has been translated into the constraint on the Higgs invisible decay as $\operatorname{Br}\left(h_{1} \rightarrow\right.$ inv $) \leq 0.11[18]$. The grey region is excluded by the perturbative unitarity bound $\lambda_{\mathrm{S}} \leq 8 \pi / 3$ [19]. The red band represents the parameter space which can reproduce the observed DM relic abundance within $3 \sigma$ range of the PLANCK Collaboration data [2] in the thermal freeze-out scenario. It has been found that since the SI cross section is suppressed

\footnotetext{
${ }^{3}$ For example, the deviation of the LO and the approximation is $9 \%$ and $-14 \%$, respectively, for $\left(m_{h_{2}}, v / v_{s}\right)=(1 \mathrm{TeV}, 0.5), \sin \theta=0.1$ and $m_{\chi}=1 \mathrm{TeV}$. Such behavior is observed in a similar DM model studied in ref. [17].
} 


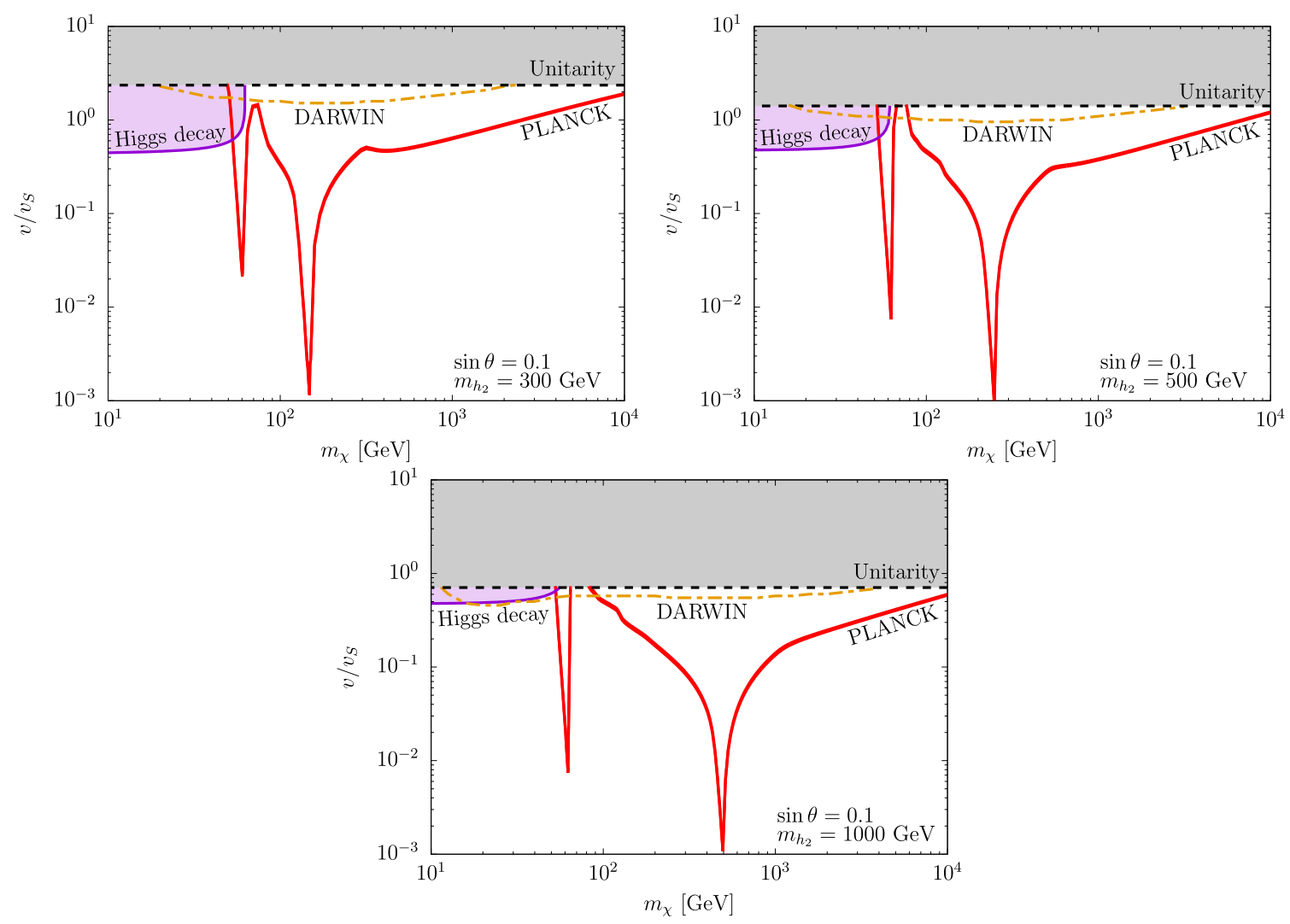

Figure 3. Exclusion limits on the model from the Higgs decay (purple) and the perturbative unitarity (grey). The red band represents the parameter space which can reproduce the observed relic abundance in the thermal freeze-out scenario. The future prospect of the DARWIN experiment is also shown as the orange dot-dashed line [9] (region above the line can be probed).

in a wide range of parameter space, there is no substantial constraint on the model from the current experimental limit of the XENON1T experiment [3]. To be concrete, the region excluded by XENON1T is always in the unitarity bound. In the plots, the future reach of the DARWIN experiment [9] is also shown in the orange dot-dashed line, assuming that all the DM abundance is composed by $\chi$. It indicates that a part of the parameter space (70 GeV $\lesssim m_{\chi} \lesssim 100 \mathrm{GeV}$ ) can be probed by the DARWIN experiment, where the thermal relic DM scenario can reproduce all the DM abundance. We have seen a similar indication for larger $\sin \theta$ values. Therefore the future direct detection experiments will be able to probe a part of the thermal freeze-out scenario with $m_{\chi} \sim 100 \mathrm{GeV}$.

\section{Conclusion}

We have studied the detectability of DM in a model where a complex singlet scalar is added to the SM. In the model, a softly broken global U(1) symmetry has been assumed, and a would-be Nambu-Goldstone boson $\chi$ becomes a candidate for DM due to a remnant $\mathbb{Z}_{2}$ symmetry. Since $\chi$ interacts with the SM particles via so-called Higgs portal coupling, it would be possible to directly detect $\chi \mathrm{DM}$ via $\chi$-nucleon scattering. It is known, however, 
that the tree-level scattering amplitude vanishes in non-relativistic limit. Thus we have taken into account the scattering process at one-loop level for non-QCD part. For QCD effect, on the other hand, the scattering amplitude has been calculated at the next-toleading order in QCD strong coupling systematically to reduce the theoretical uncertainties. It has been found that the predicted SI cross section is so small in a wide range of the parameter space of the model that there is no substantial bound from the current direct detection experiments. However, a part of the parameter space, which includes canonical thermal relic scenario accounting for the present DM abundance, will be probed in the future direct detection experiment DARWIN.

\section{Acknowledgments}

This work was supported by JSPS KAKENHI Grant Numbers JP17H05402, JP17K14278, JP17H02875 and JP18H05542 (K.I.). T.T. acknowledges support from JSPS Fellowships for Research Abroad.

Note added. While completing our paper, we found that ref. [21] studied the direct detection of dark matter in the same model. We agree qualitatively with their results in large dark matter mass region, as well as in the other dark matter mass region if the diagrams (iii) are ignored. On the other hand, we have seen a different behavior in low dark matter mass region. This is due to the diagrams (iii), which are discarded in their study. Although these diagrams are irrelevant for the estimation of the current bound from the XENON1T experiment, it will be important for the future study of this model in the direct detection experiments.

\section{A Loop functions}

The loop functions are basically expressed by so-called $A_{0}$ function and $B_{0}$ function (and their derivatives),

$$
\begin{aligned}
i A_{0}\left(m^{2}\right) & =\int \frac{d^{D} q}{(2 \pi)^{D}} \frac{1}{q^{2}-m^{2}}, \\
i B_{0}\left(p^{2} ; m_{1}^{2}, m_{2}^{2}\right) & =\int \frac{d^{D} q}{(2 \pi)^{D}} \frac{1}{\left(q^{2}-m_{1}^{2}\right)\left((q+p)^{2}-m_{2}^{2}\right)} . \\
i C^{i j} & =\int \frac{d^{D} q}{(2 \pi)^{D}} \frac{1}{\left((q+P)^{2}-m_{\chi}^{2}\right)\left(q^{2}-m_{h_{i}}^{2}\right)\left(q^{2}-m_{h_{j}}^{2}\right)}, \\
i C^{\chi i} & =\int \frac{d^{D} q}{(2 \pi)^{D}} \frac{1}{\left((q+P)^{2}-m_{h_{i}}^{2}\right)\left(q^{2}-m_{\chi}^{2}\right)\left(q^{2}-m_{\chi}^{2}\right)},
\end{aligned}
$$

where $P^{2}=m_{\chi}^{2}$ in the last two functions. Here the divergent pieces are subtracted in the $\overline{\mathrm{MS}}$ renormalization scheme implicitly. We use the analytic expressions for the loop functions, which are numerically checked by using LoopTools [20]. 


\section{A.1 Tadpoles}

The tadpoles $T_{h_{i}}$ are given by $A_{0}$ function as,

$$
\begin{aligned}
& T_{h_{1}}=3 c_{h_{1} h_{1} h_{1}} A_{0}\left(m_{h_{1}}^{2}\right)+c_{h_{1} h_{2} h_{2}} A_{0}\left(m_{h_{2}}^{2}\right)+c_{\chi \chi h_{1}} A_{0}\left(m_{\chi}^{2}\right)-y_{t t h_{1}} m_{t} A_{0}\left(m_{t}^{2}\right), \\
& T_{h_{2}}=3 c_{h_{2} h_{2} h_{2}} A_{0}\left(m_{h_{2}}^{2}\right)+c_{h_{1} h_{1} h_{2}} A_{0}\left(m_{h_{1}}^{2}\right)+c_{\chi \chi h_{2}} A_{0}\left(m_{\chi}^{2}\right)-y_{t t h_{2}} m_{t} A_{0}\left(m_{t}^{2}\right) .
\end{aligned}
$$

Then $T_{h}$ and $T_{s}$ are obtained by rotating with the orthogonal matrix $O$ given in eq. (2.18) as $\left(T_{h}, T_{s}\right)^{T}=O\left(T_{h_{1}}, T_{h_{2}}\right)^{T}$.

\section{A.2 Self-energies}

The one-loop contributions to the self-energies $\Pi_{h_{1} h_{1}}, \Pi_{h_{2} h_{2}}$, and $\Pi_{h_{1} h_{2}}$ are listed below:

$$
\begin{aligned}
& \Pi_{h_{1} h_{1}}\left(p^{2}\right)=2 c_{\chi \chi h_{1}}^{2} B_{0}\left(p^{2} ; m_{\chi}^{2}, m_{\chi}^{2}\right)+6 c_{G^{0} G^{0} h_{1}}^{2} B_{0}\left(p^{2} ; 0,0\right) \\
& +18 c_{h_{1} h_{1} h_{1}}^{2} B_{0}\left(p^{2} ; m_{h_{1}}^{2}, m_{h_{1}}^{2}\right)+2 c_{h_{1} h_{2} h_{2}}^{2} B_{0}\left(p^{2} ; m_{h_{2}}^{2}, m_{h_{2}}^{2}\right)+4 c_{h_{1} h_{1} h_{2}}^{2} B_{0}\left(p^{2} ; m_{h_{1}}^{2}, m_{h_{2}}^{2}\right) \\
& +2 d_{\chi \chi h_{1} h_{1}} A_{0}\left(m_{\chi}^{2}\right)+12 d_{h_{1} h_{1} h_{1} h_{1}} A_{0}\left(m_{h_{1}}^{2}\right)+2 d_{h_{1} h_{1} h_{2} h_{2}} A_{0}\left(m_{h_{2}}^{2}\right) \\
& -4 y_{t t h_{1}}^{2}\left(A_{0}\left(m_{t}^{2}\right)+\left(2 m_{t}^{2}-p^{2} / 2\right) B_{0}\left(p^{2} ; m_{t}^{2}, m_{t}^{2}\right)\right) \text {, } \\
& \Pi_{h_{2} h_{2}}\left(p^{2}\right)=2 c_{\chi \chi h_{2}}^{2} B_{0}\left(p^{2} ; m_{\chi}^{2}, m_{\chi}^{2}\right)+6 c_{G^{0} G^{0} h_{2}}^{2} B_{0}\left(p^{2} ; 0,0\right) \\
& +18 c_{h_{2} h_{2} h_{2}}^{2} B_{0}\left(p^{2} ; m_{h_{2}}^{2}, m_{h_{2}}^{2}\right)+2 c_{h_{1} h_{1} h_{2}}^{2} B_{0}\left(p^{2} ; m_{h_{1}}^{2}, m_{h_{1}}^{2}\right)+4 c_{h_{1} h_{2} h_{2}}^{2} B_{0}\left(p^{2} ; m_{h_{1}}^{2}, m_{h_{2}}^{2}\right) \\
& +2 d_{\chi \chi h_{2} h_{2}} A_{0}\left(m_{\chi}^{2}\right)+12 d_{h_{2} h_{2} h_{2} h_{2}} A_{0}\left(m_{h_{2}}^{2}\right)+2 d_{h_{1} h_{1} h_{2} h_{2}} A_{0}\left(m_{h_{1}}^{2}\right) \\
& -4 y_{t t h_{2}}^{2}\left(A_{0}\left(m_{t}^{2}\right)+\left(2 m_{t}^{2}-p^{2} / 2\right) B_{0}\left(p^{2} ; m_{t}^{2}, m_{t}^{2}\right)\right) \text {, } \\
& \Pi_{h_{1} h_{2}}\left(p^{2}\right)=2 c_{\chi \chi h_{1}} c_{\chi \chi h_{2}} B_{0}\left(p^{2} ; m_{\chi}^{2}, m_{\chi}^{2}\right)+6 c_{G^{0} G^{0} h_{1}} c_{G^{0} G^{0} h_{2}} B_{0}\left(p^{2} ; 0,0\right) \\
& +6 c_{h_{1} h_{1} h_{1}} c_{h_{1} h_{1} h_{2}} B_{0}\left(p^{2} ; m_{h_{1}}^{2}, m_{h_{1}}^{2}\right)+6 c_{h_{2} h_{2} h_{2}} c_{h_{1} h_{2} h_{2}} B_{0}\left(p^{2} ; m_{h_{2}}^{2}, m_{h_{2}}^{2}\right) \\
& +4 c_{h_{1} h_{1} h_{2}} c_{h_{1} h_{2} h_{2}} B_{0}\left(p^{2} ; m_{h_{1}}^{2}, m_{h_{2}}^{2}\right) \\
& +d_{\chi \chi h_{1} h_{2}} A_{0}\left(m_{\chi}^{2}\right)+3 d_{h_{1} h_{1} h_{1} h_{2}} A_{0}\left(m_{h_{1}}^{2}\right)+3 d_{h_{1} h_{2} h_{2} h_{2}} A_{0}\left(m_{h_{2}}^{2}\right) \\
& -4 y_{t t h_{1}} y_{t t h_{2}}\left(A_{0}\left(m_{t}^{2}\right)+\left(2 m_{t}^{2}-p^{2} / 2\right) B_{0}\left(p^{2} ; m_{t}^{2}, m_{t}^{2}\right)\right) \text {. }
\end{aligned}
$$

Then, $\Pi_{h h}, \Pi_{s s}$, and $\Pi_{h s}$ are obtained similarly to eq. (2.17).

\section{A.3 Vertex corrections}

The corrections to the cubic couplings $c_{\chi \chi} h_{i}$ are given by

$$
\Delta c_{\chi \chi h_{i}}=\Delta c_{\chi \chi h_{i}}^{\mathrm{c}}+\Delta c_{\chi \chi h_{i}}^{\mathrm{t}},
$$




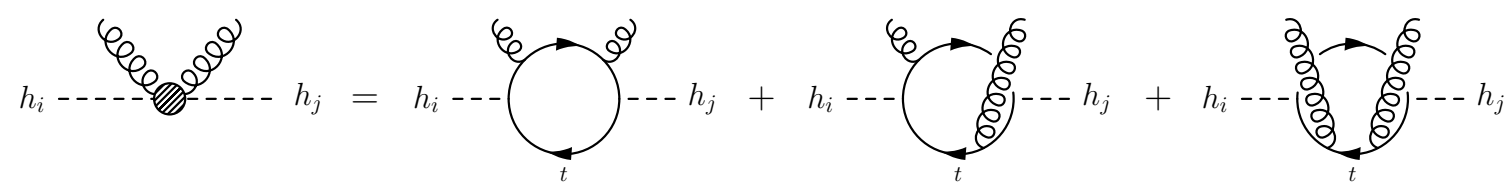

Figure 4. Higgs correlation functions.

with

$$
\begin{aligned}
& -\Delta c_{\chi \chi h_{1}}^{\mathrm{c}}=6 d_{\chi \chi h_{1} h_{1}} c_{h_{1} h_{1} h_{1}} B_{0}\left(0 ; m_{h_{1}}^{2}, m_{h_{1}}^{2}\right)+2 d_{\chi \chi h_{1} h_{2}} c_{h_{1} h_{1} h_{2}} B_{0}\left(0 ; m_{h_{1}}^{2}, m_{h_{2}}^{2}\right) \\
& +2 d_{\chi \chi h_{2} h_{2}} c_{h_{1} h_{2} h_{2}} B_{0}\left(0 ; m_{h_{2}}^{2}, m_{h_{2}}^{2}\right)+12 d_{\chi \chi \chi \chi} c_{\chi \chi h_{1}} B_{0}\left(0 ; m_{\chi}^{2}, m_{\chi}^{2}\right) \\
& +8 d_{\chi \chi h_{1} h_{1}} c_{\chi \chi h_{1}} B_{0}\left(0 ; m_{\chi}^{2}, m_{h_{1}}^{2}\right)+4 d_{\chi \chi h_{1} h_{2}} c_{\chi \chi h_{2}} B_{0}\left(0 ; m_{\chi}^{2}, m_{h_{2}}^{2}\right), \\
& -\Delta c_{\chi \chi h_{2}}^{\mathrm{c}}=6 d_{\chi \chi h_{2} h_{2}} c_{h_{2} h_{2} h_{2}} B_{0}\left(0 ; m_{h_{2}}^{2}, m_{h_{2}}^{2}\right)+2 d_{\chi \chi h_{1} h_{2}} c_{h_{1} h_{2} h_{2}} B_{0}\left(0 ; m_{h_{1}}^{2}, m_{h_{2}}^{2}\right) \\
& +2 d_{\chi \chi h_{1} h_{1}} c_{h_{1} h_{1} h_{2}} B_{0}\left(0 ; m_{h_{2}}^{2}, m_{h_{2}}^{2}\right)+12 d_{\chi \chi \chi \chi} c_{\chi \chi h_{2}} B_{0}\left(0 ; m_{\chi}^{2}, m_{\chi}^{2}\right) \\
& +8 d_{\chi \chi h_{2} h_{2}} c_{\chi \chi h_{2}} B_{0}\left(0 ; m_{\chi}^{2}, m_{h_{2}}^{2}\right)+4 d_{\chi \chi h_{1} h_{2}} c_{\chi \chi h_{1}} B_{0}\left(0 ; m_{\chi}^{2}, m_{h_{1}}^{2}\right) \text {, } \\
& -\Delta c_{\chi \chi h_{1}}^{\mathrm{t}}=12 c_{\chi \chi h_{1}}^{2} c_{h_{1} h_{1} h_{1}} C^{11}+8 c_{\chi \chi h_{1}} c_{\chi \chi h_{2}} c_{h_{1} h_{1} h_{2}} C^{12}+4 c_{\chi \chi h_{2}}^{2} c_{h_{1} h_{2} h_{2}} C^{22} \\
& +4 c_{\chi \chi h_{1}}^{3} C^{\chi 1}+4 c_{\chi \chi h_{1}} c_{\chi \chi h_{2}}^{2} C^{\chi^{2}} \text {, } \\
& -\Delta c_{\chi \chi h_{2}}^{\mathrm{t}}=12 c_{\chi \chi h_{2}}^{2} c_{h_{2} h_{2} h_{2}} C^{22}+8 c_{\chi \chi h_{1}} c_{\chi \chi h_{2}} c_{h_{1} h_{2} h_{2}} C^{12}+4 c_{\chi \chi h_{1}}^{2} c_{h_{1} h_{1} h_{2}} C^{11} \\
& +4 c_{\chi \chi h_{2}}^{3} C^{\chi^{2}}+4 c_{\chi \chi h_{1}}^{2} c_{\chi \chi h_{2}} C^{\chi^{1}} \text {. }
\end{aligned}
$$

As described in the main text of the paper, the renormalization scale dependence is cancelled in the Wilson coefficients, $\left.C_{\mathrm{S}}^{q}\left(\mu_{W}\right)\right|_{\text {self }}+\left.C_{\mathrm{S}}^{q}\left(\mu_{W}\right)\right|_{\text {vert }}$.

It would be helpful to see how it behaves in small and large $m_{\chi}$ limit. For $m_{\chi} \rightarrow 0$ limit, we have found that it is proportional to $m_{\chi}^{2}$. For example, ${ }^{4}$

$$
\begin{aligned}
\left.C_{\mathrm{S}}^{q}\left(\mu_{W}\right)\right|_{\text {self }}+\left.C_{\mathrm{S}}^{q}\left(\mu_{W}\right)\right|_{\text {vert }} \\
\quad \rightarrow\left\{\begin{array}{ll}
\frac{2 m_{\chi}^{2} m_{h_{2}}^{4}}{v v_{s}^{2}} \sin \theta \cos ^{4} \theta\left(v \cos \theta-v_{s} \sin \theta\right) & \left(\text { for } m_{h_{1}}=0\right) \\
-\frac{2 m_{\chi}^{2} m_{h_{1}}^{4}}{v v_{s}^{2}} \sin ^{4} \theta \cos \theta\left(v \sin \theta+v_{s} \cos \theta\right) & \left(\text { for } m_{h_{2}}=0\right)
\end{array} .\right.
\end{aligned}
$$

In large $m_{\chi}$ limit, on the other hand, its absolute value increases logarithmically, e.g.,

$$
\begin{aligned}
& \left.C_{\mathrm{S}}^{q}\left(\mu_{W}\right)\right|_{\text {self }}+\left.C_{\mathrm{S}}^{q}\left(\mu_{W}\right)\right|_{\text {vert }} \\
& \quad \rightarrow\left\{\begin{array}{cl}
\frac{2\left[\log \left(m_{\chi}^{2} / m_{h_{2}}^{2}\right)+2\right] m_{h_{2}}^{6}}{v v_{s}^{2}} \sin \theta \cos ^{4} \theta\left(v \cos \theta-v_{s} \sin \theta\right) & \left(\text { for } m_{h_{1}}=0\right) \\
-\frac{2\left[\log \left(m_{\chi}^{2} / m_{h_{1}}^{2}\right)+2\right] m_{h_{1}}^{6}}{v v_{s}^{2}} \sin ^{4} \theta \cos \theta\left(v \sin \theta+v_{s} \cos \theta\right) & \left(\text { for } m_{h_{2}}=0\right)
\end{array} .\right.
\end{aligned}
$$

\section{A.4 Box and triangle diagrams}

To compute the Wilson coefficients induced by $\chi-g$ scattering, it is legitimate to derive Higgs correlation functions shown in figure 4 . We denote them as $\tilde{\Pi}_{i j}\left(q^{2}\right)$, which are obtained straightforwardly by using the formula given in refs. $[11,12]$ as,

$$
\tilde{\Pi}_{i j}\left(q^{2}\right)=c^{G}\left(q^{2} ; \mu_{W}\right) \frac{\alpha_{s}}{\pi} G_{\mu \nu}^{a} G^{a \mu \nu},
$$

\footnotetext{
${ }^{4}$ We omit the expression for non-zero $m_{h_{1}}$ and $m_{h_{2}}$ since it is too lengthy. (It is similar for large $m_{\chi}$ case.)
} 
where

$$
c^{G}\left(q^{2} ; \mu_{W}\right)=-\frac{c_{q q h_{i}} c_{q q h_{j}}}{8 m_{\chi}^{2}} I\left(-q^{2} / m_{\chi}^{2}, x_{t}\right) .
$$

Here $I\left(t, x_{t}\right)$ is a dimensionless function,

$$
I\left(t, x_{t}\right)=\frac{t-2 x_{t}}{t\left(t+4 x_{t}\right)}+\frac{2 x_{t}\left(t+x_{t}\right)}{t^{2}\left(t+4 x_{t}\right) \beta\left(t, x_{t}\right)} \log \left[\frac{2 x_{t}+t\left(1+\beta\left(t, x_{t}\right)\right)}{2 x_{t}+t\left(1-\beta\left(t, x_{t}\right)\right)}\right],
$$

with $\beta\left(t, x_{t}\right)=\sqrt{1+4 x_{t} / t}, x_{i}=m_{h_{i}}^{2} / m_{\chi}^{2}$, and $x_{t}=m_{t}^{2} / m_{\chi}^{2}$. Then, the Wilson coefficients coming from the box and triangle diagrams are given by the following integrals,

$$
\begin{aligned}
J_{\text {box }}^{i j} & =\frac{\kappa^{i j}}{8(4 \pi)^{2}} \int_{0}^{\infty} d t \frac{t I\left(t, x_{t}\right)}{\left(t+x_{i}\right)\left(t+x_{j}\right)}(1-\sqrt{(t+4) / t}), \\
J_{\text {tri }}^{i j} & =\frac{1}{8(4 \pi)^{2}} \int_{0}^{\infty} d t \frac{t I\left(t, x_{t}\right)}{\left(t+x_{i}\right)\left(t+x_{j}\right)},
\end{aligned}
$$

with $\kappa^{i j}=2$ for $i \neq j$ otherwise 1 . As mentioned in the main text of the paper, $J_{\mathrm{tri}}^{i j} / m_{\chi}^{2}$ is constant with respect to $m_{\chi}$. On the other hand, $J_{\text {box }}^{i j} \propto m_{\chi}^{4}\left(m_{\chi}^{3}\right)$ for small (large) $m_{\chi}$ region. Therefore the contribution from the box diagrams is suppressed as $1 / m_{\chi}$ in large DM mass region while it becomes constant in small DM mass region.

Open Access. This article is distributed under the terms of the Creative Commons Attribution License (CC-BY 4.0), which permits any use, distribution and reproduction in any medium, provided the original author(s) and source are credited.

\section{References}

[1] Planck collaboration, N. Aghanim et al., Planck 2018 results. VI. Cosmological parameters, arXiv: 1807.06209 [INSPIRE].

[2] Planck collaboration, P.A.R. Ade et al., Planck 2015 results. XIII. Cosmological parameters, Astron. Astrophys. 594 (2016) A13 [arXiv: 1502.01589] [INSPIRE].

[3] XENON collaboration, E. Aprile et al., Dark matter search results from a one ton-year exposure of XENON1 T, Phys. Rev. Lett. 121 (2018) 111302 [arXiv:1805.12562] [INSPIRE].

[4] C. Gross, O. Lebedev and T. Toma, Cancellation mechanism for dark-matter-nucleon interaction, Phys. Rev. Lett. 119 (2017) 191801 [arXiv:1708.02253] [INSPIRE].

[5] A. Berlin, S. Gori, T. Lin and L.-T. Wang, Pseudoscalar portal dark matter, Phys. Rev. D 92 (2015) 015005 [arXiv: 1502.06000] [INSPIRE].

[6] G. Arcadi, M. Lindner, F.S. Queiroz, W. Rodejohann and S. Vogl, Pseudoscalar mediators: a WIMP model at the neutrino floor, JCAP 03 (2018) 042 [arXiv:1711.02110] [INSPIRE].

[7] T. Abe, M. Fujiwara and J. Hisano, Loop corrections to dark matter direct detection in a pseudoscalar mediator dark matter model, arXiv:1810.01039 [INSPIRE].

[8] J. Hisano, K. Ishiwata and N. Nagata, QCD effects on direct detection of Wino dark matter, JHEP 06 (2015) 097 [arXiv: 1504.00915] [INSPIRE].

[9] DARWIN collaboration, J. Aalbers et al., DARWIN: towards the ultimate dark matter detector, JCAP 11 (2016) 017 [arXiv:1606.07001] [INSPIRE]. 
[10] A. Brignole, Radiative corrections to the supersymmetric neutral Higgs boson masses, Phys. Lett. B 281 (1992) 284 [INSPIRE].

[11] J. Hisano, K. Ishiwata and N. Nagata, A complete calculation for direct detection of Wino dark matter, Phys. Lett. B 690 (2010) 311 [arXiv:1004.4090] [INSPIRE].

[12] J. Hisano, K. Ishiwata and N. Nagata, Gluon contribution to the dark matter direct detection, Phys. Rev. D 82 (2010) 115007 [arXiv:1007.2601] [INSPIRE].

[13] J. Hisano, R. Nagai and N. Nagata, Effective theories for dark matter nucleon scattering, JHEP 05 (2015) 037 [arXiv: 1502.02244] [INSPIRE].

[14] R.D. Young and A.W. Thomas, Octet baryon masses and sigma terms from an $\mathrm{SU}(3)$ chiral extrapolation, Phys. Rev. D 81 (2010) 014503 [arXiv:0901.3310] [INSPIRE].

[15] JLQCD collaboration, H. Ohki et al., Nucleon strange quark content from $N_{f}=2+1$ lattice QCD with exact chiral symmetry, Phys. Rev. D 87 (2013) 034509 [arXiv:1208.4185] [INSPIRE].

[16] J.F. Owens, A. Accardi and W. Melnitchouk, Global parton distributions with nuclear and finite- $Q^{2}$ corrections, Phys. Rev. D 87 (2013) 094012 [arXiv: 1212.1702] [INSPIRE].

[17] K. Endo and K. Ishiwata, Direct detection of singlet dark matter in classically scale-invariant standard model, Phys. Lett. B 749 (2015) 583 [arXiv:1507.01739] [INSPIRE].

[18] ATLAS and CMS collaborations, Measurements of the Higgs boson production and decay rates and constraints on its couplings from a combined ATLAS and CMS analysis of the LHC pp collision data at $\sqrt{s}=7$ and $8 \mathrm{TeV}$, JHEP 08 (2016) 045 [arXiv: 1606.02266] [INSPIRE].

[19] C.-Y. Chen, S. Dawson and I.M. Lewis, Exploring resonant di-Higgs boson production in the Higgs singlet model, Phys. Rev. D 91 (2015) 035015 [arXiv:1410.5488] [INSPIRE].

[20] T. Hahn and M. Pérez-Victoria, Automatized one loop calculations in four-dimensions and D-dimensions, Comput. Phys. Commun. 118 (1999) 153 [hep-ph/9807565] [InSPIRE].

[21] D. Azevedo, M. Duch, B. Grzadkowski, D. Huang, M. Iglicki and R. Santos, One-loop contribution to dark matter-nucleon scattering in the pseudoscalar dark matter model, arXiv: 1810.06105 [INSPIRE]. 